\title{
Lipid A-Ara4N as an alternate pathway for (colistin) resistance in Klebsiella pneumonia isolates in Pakistan
}

\author{
Kiran lqbal Masood', Seema Umar', Zahra Hasan', Joveria Farooqi', Safina Abdul Razzak', Nazish Jabeen', \\ Jason Rao ${ }^{3}$, Sadia Shakoor ${ }^{1}$ and Rumina Hasan ${ }^{1,2^{*}}$ (B)
}

\begin{abstract}
Objectives: This study aimed to explore mechanism of colistin resistance amongst Klebsiella pneumoniae isolates through plasmid mediated mcr-1 gene in Pakistan. Carbapenem and Colistin resistant K. pneumoniae isolates $(n=34)$ stored at $-80^{\circ} \mathrm{C}$ as part of the Aga Khan University Clinical Laboratory strain bank were randomly selected and subjected to mcr-1 gene PCR. To investigate mechanisms of resistance, other than plasmid mediated mcr-1 gene, whole genome sequencing was performed on 8 clinical isolates, including 6 with colistin resistance $(\mathrm{MIC}>4 \mu \mathrm{g} / \mathrm{ml}$ ) and 2 with intermediate resistance to colistin (MIC $>2 \mu \mathrm{g} / \mathrm{ml}$ ).

Results: RT-PCR conducted revealed absence of $\mathrm{mcr}^{-1}$ gene in all isolates tested. Whole genome sequencing results revealed modifications in Lipid A-Ara4N pathway. Modifications in Lipid A-Ara4N pathway were detected in ArnA_DH/ FT, UgdH, ArnC and ArnT genes. Mutation in ArnA_DH/FT gene were detected in S3, S5, S6 and S7 isolates. UgdH gene modifications were found in all isolates except S3, mutations in ArnC were present in all except S1, S2 and S8 and ArnT were detected in all except S4 and S7. In the absence of known mutations linked with colistin resistance, lipid pathway modifications may possibly explain the phenotype resistance to colistin, but this needs further exploration.
\end{abstract}

Keywords: Klebsiella pneumonia, mcr-1 gene, Antimicrobial drug resistance

\section{Introduction}

Beta-lactams have always been the backbone of antibiotic regimens targeting infections with Enterobacteriaceae including $K$. pneumoniae [1]. With the increase in resistance to these agents $[1,2]$ there is interest in using colistin (polymyxin E) for treating such infections. Colistin targets lipopolysaccharides (LPS) and phospholipids present in the outer cell membrane of Gram-negative bacteria, and competitively displaces divalent cations from the phosphate groups of membrane lipids leading to pore

\footnotetext{
*Correspondence: rumina.hasan@aku.edu

1 Department of Pathology and Laboratory Medicine, Aga Khan

University, Karachi, Pakistan

Full list of author information is available at the end of the article
}

formation and disruption of the outer cell membrane, leakage of cytoplasmic contents, and bacterial death.

A worldwide increase in resistance to colistin has been reported globally $[3,4]$. Such resistance can either be acquired, or occur as a result of intrinsic factors [5]. Mobile colistin resistance $(m c r)$ genes acquired through plasmids were first reported in China in 2015 [6]. The first colistin resistant $E$. coli with $m c r-1$ was reported from Pakistan in 2017 [7].

The intrinsic factors contributing to colistin resistance mainly involves the modification of the LPS moiety by the addition of positively charged molecules L-Ara4-N and PEtN [8]. The addition of positively-charged molecules result in the decrease of negative charge in the outer membrane thus reducing its affinity to interact with cationic antibiotics including colistin [9]. Alterations in 
LPS most commonly occur due to mutations in bacterial genes including $\mathrm{mgrB}$, phoP/phoQ, pmrA, pmrB, pmrC, and crrABC [10-12].Increasing colistin resistance amongst $K$. pneumoniae emphasizes the need to understand mechanisms contributing to such resistance. This study is therefore aimed to explore mechanism of colistin resistance amongst $K$. pneumoniae isolates from Pakistan, and to study the role of plasmid-mediated ( $m c r-1$ gene) and chromosomal factors contributing to such resistance.

\section{Main text \\ Methods}

\section{Study subjects}

The clinical isolates $(n=34)$ were randomly selected from the Aga Khan University Clinical Laboratory strain bank stored at $-80{ }^{\circ} \mathrm{C}$ and used anonymously. The source of the selected strains included sputum (n:1), tracheal aspirates (n:9), blood (n:9), urine (n:8), tissue (n:4), pus (n:2) and cerebrospinal fluid (n:1). All the study isolates were pure and were not passaged for more than 3 times.

\section{Identification of bacterial isolates and susceptibility testing}

The isolates were identified by conventional biochemical methods followed by API-E ${ }^{\circledR}$ (BioMérieux, France). Susceptibilities performed by disc diffusion (Kirby-Bauer) method on Mueller Hinton agar [13] and VITEK2 ${ }^{\circledR}$ system (BioMérieux, France) showed resistance against beta-lactams and carbapenems (imipenem/meropenem).

Colistin susceptibility was performed using broth micro-dilution according to Clinical and Laboratory Standards Institute (CLSI) guidelines, M07-A10, 2019 [14]. Minimum Inhibitory Concentrations (MIC) were performed in 96-well polystyrene microtitre plates using Cation-Adjusted Mueller Hinton Broth and colistin sulfate powders (Sigma-Aldrich, Inc.) Colistin concentration of $0.03-16.0 \mu \mathrm{g} / \mathrm{ml}$ was used [15]. ATCC E. coli 25922, ATCC P. aeruginosa 27853 and NCTC E. coli 13486 were used as controls. Results were read and interpreted after $16-20 \mathrm{~h}$ at $35^{\circ} \mathrm{C}$ using colistin cut-offs $(\leq 2 \mu \mathrm{g} / \mathrm{ml}$ $=$ intermediate; $\geq 4 \mu \mathrm{g} / \mathrm{ml}=$ resistant) for Enterobacteriales [14].

\section{DNA extraction}

The selected isolates $(\mathrm{n}=34)$ were inoculated into Brain heart infusion for $24 \mathrm{~h} .1 \mathrm{ml}$ of the broth was used for DNA extraction as per QIA $^{\circledR}$ amp DNA extraction kit (QIAGEN, USA) manufacturer's protocol. The quality of the extracted DNA was examined using Qubit v.2.0 fluorometer (Life Technologies, USA). The extracted DNA was stored at $-80^{\circ} \mathrm{C}$ till further processing.

\section{mcr-1 gene real-time PCR}

Real-time PCR was performed on the extracted DNA in duplicate. $20-\mu$ l reactions were set up containing Platinum ${ }^{\circledR}$ SYBR $^{\circledR}$ Green qPCR Supermix-UDG (Invitrogen), $150 \mathrm{~nm}$ forward and reverse primers and $2 \mu \mathrm{l}$ of DNA on a BioRad CFX 7500 thermal cycler. Sequence specific primers were used for $m c r-1$ [16] gene and the house keeping gene rho, [17]. Primer sequences are provided in Additional file 1: Table S1. NCTC 13846 E. coli was used as positive control.

\section{Whole genome sequencing}

Eight $K$. pneumoniae (CRKP1-CRKP6) strains were selected for whole genome sequencing (WGS) with six resistant and two intermediate to colistin. We had clinical data on only 4 isolates: one colistin intermediate isolate failed microbiological clearance for 3 years despite treatment, while three colistin resistant isolates achieved microbiological clearance between 7 and 11 days.

DNA was shipped to Eurofins scientific SE, Luxembourg for sequencing. Sequencing was carried out on the Illumina NovaSeq 6000 platform using $2 \times 150$ Sequence mode. WGS was performed with the genome coverage of $99 \%$. Genetic analysis of reads was done using the KmerGeni tool which generated an approximate of 5.1 Mbp contigs. The de novo assemblies were evaluated by QUAST and Icarus tools. The evaluation was based on the alignment of the de novo assemblies on the reference genome (HS11286). The contigs were annotated with RAST to look for resistance genes.

\section{Data submission}

The raw sequences generated after sequencing were submitted to NCBI-SRA website under the accession numbers SAMN16684225-SAMN16684230. This Whole Genome Shotgun project genome assemblies were deposited at DDB/ENA/GenBank under the accession JAEMV $(\mathrm{N} / \mathrm{O} / \mathrm{P} / \mathrm{Q} / \mathrm{R} / \mathrm{S}) 000000000$. The version described is JAEMVX010000000. The complete data details are available in the BioProject PRJNA674952.

\section{Data analysis}

The raw Illumina reads were passed through quality check using FASTQC and then assembled with SPAdes-3.13.0 software using spade and plasmid spade scripts to generate raw chromosomal and plasmid contigs. The generated assembly quality assessment was conducted using QUAST (http://bioinf.spbau.ru/quast). Continuous chromosome sequence was generated by overlapping raw contigs assembly against the reference genome by abacas.1.3.1 perl script that closed gaps on shotgun assembled contigs against the reference genome 
based on alignment between assembly and reference to identify syntenies of contigs with the reference.

Multi-Locus Sequence Typing (MLST) was determined using K. pneumoniae Sequence Typing web-based tool (PasteurMLST) (https://bigsdb.pasteur.fr/). The MLST was performed using the seven housekeeping genes (gapA, infB, mdh, pgi, phoE, rpoB and tonB) according to the protocol described by Diancourt et al. [18]. RAST server (https://rast.nmpdr.org/) and Center for Genomic Epidemiology server (www.cbs.dtu.dk/services) were used for Chromosomal and Plasmid sequence annotations and downstream analysis.

\section{Phylogenetic analysis}

The phylogenetic inference was done by aligning the eight isolates genome with the reference using MegaX software (https://www.megasoftware.net/). The aligned sequences were then converted into a Phylodendogram using the software package Clonal Frame version 1.1. The dendogram was then estimated under the maximum likelihood (ML) principle in PhyloXML (http://www.phylo xml.org/). The tree was colored and edited using FigTree (http://tree.bio.ed.ac.uk/software/figtree/). The tree was further magnified to show closely related genome using SNP (single nucleotide polymorphism) cluster generated by Pathogen detection Browser (https://www.ncbi.nlm. nih.gov/pathogens/isolates/).

\section{Results}

\section{mcr-1 gene PCR}

A total of 34 colistin resistant $K$. pneumoniae isolates were included. These strains were investigated for the presence of $m c r-1$ gene. However, PCR conducted revealed absence of $m c r-1$ gene in all isolates tested.

\section{Alternate mechanisms involved in colistin resistance}

WGS was performed to further understand the mechanism of colistin resistance. Mutations in genes involved in lipid-A and Ara-4 N pathways were revealed through a variant analysis of non-synonymous single nucleotide variants (ns-SNVs Table 1).

The mutations detected in PagP gene (I189F) were present in all isolates. In Pho $\mathrm{R}$ gene, mutations identified included: A424_V425insSerAla present in all isolates except S3, S4 and S8; L65F present in all isolates except S2 and S7. Additionally, M45I mutation was also identified in isolate S7.

Modifications in Lipid A-Ara4N pathway were detected in ArnA_DH/FT, UgdH, ArnC and ArnT genes. ArnA_DH/FT included mutations T185A, S18A, L260I and D205N. Mutations detected in $U g d H$ were N354D, V17I, N354D. Mutation detected in ArnC was S10fs and in $A r n T$ was A55G.

Mutations in ArnA_DH/FT gene were detected in S3, $\mathrm{S} 5, \mathrm{~S} 6$ and $\mathrm{S} 7$ isolates. $U g d H$ gene modification was found in all isolates except S3, mutations in ArnC were present in all except S1, S2 and S8 and ArnT were detected in all except S4 and S7.

\section{Multi locus sequence typing of K. pneumoniae}

MLST revealed that the isolates belonged to ST37 $(n=1)$, ST147 $(n=3)$, ST14 $(n=1)$, ST $11(n=1)$, ST39 $(n=1)$ and ST $17(n=1)$ sequence types (Table 2$)$.

Table 1 Lipid A pathways related variants identified in colistin-resistant Klebsiella pneumoniae isolates

\begin{tabular}{|c|c|c|c|c|c|c|c|c|}
\hline \multirow[t]{2}{*}{ Isolates } & \multirow[t]{2}{*}{ MIC } & \multirow[t]{2}{*}{ GenBank accession } & \multicolumn{2}{|c|}{ Lipid A modification } & \multicolumn{4}{|c|}{ Lipid A-Ara4N pathway (polymyxin resistance) } \\
\hline & & & PagP & PhoR & ArnA_DH/FT & $U g d H$ & ArnC & ArnT \\
\hline S1 & 0.25 & JAEMVQ000000000.1 & I189F & $\begin{array}{l}\text { A424_V425insSerAla } \\
\text { L65F }\end{array}$ & - & N354D & - & A55G \\
\hline S2 & 0.5 & JAEMVP000000000.1 & I189F & A424_V425insSerAla & - & V17l & S10fs & A55G \\
\hline S3 & 4 & JAEMVA000000000 & I189F & L65F & $\mathrm{T} 185 \mathrm{~A}$ & - & - & A55G \\
\hline S4 & 8 & JAEMVS000000000.1 & I189F & L65F & - & N354D & S10fs & - \\
\hline S5 & 4 & JAEMVRO00000000.1 & I189F & $\begin{array}{l}\text { A424_V425insSerAla } \\
\text { L65F }\end{array}$ & $\begin{array}{l}\text { T185A } \\
\text { S18A }\end{array}$ & N354D & $\begin{array}{l}\text { S10fs } \\
\text { S10fs }\end{array}$ & A55G \\
\hline S6 & 16 & JAEMVO000000000.1 & I189F & $\begin{array}{l}\text { A424_V425insSerAla } \\
\text { L65F }\end{array}$ & $\begin{array}{l}\mathrm{T} 185 \mathrm{~A} \\
\mathrm{~S} 18 \mathrm{~A}\end{array}$ & N354D & S10fs & A55G \\
\hline S7 & 16 & JAEMVN000000000.1 & I189F & $\begin{array}{l}\text { A424_V425insSerAla } \\
\text { M45I }\end{array}$ & $\begin{array}{l}\text { L260l } \\
\text { D205N }\end{array}$ & $\begin{array}{l}\text { A376V } \\
\text { N354D }\end{array}$ & S10fs & - \\
\hline S8 & $\geq 16$ & JAEMVB000000000 & I189F & L65F & - & V17l & - & A55G \\
\hline
\end{tabular}

MIC minimal inhibitory concentrations. The table represents various mutations (amino acid) found upon whole genome sequencing analysis of eight Klebsiella pneumonia strains isolated from clinical samples 
Table 2 The general genetic characteristics of the Klebsiella pneumoniae strains

\begin{tabular}{|c|c|c|c|c|c|c|c|}
\hline Samples & No. of contigs & $\begin{array}{l}\text { Total length } \\
\text { of assembly }\end{array}$ & N50 & GC content (\%) & $\begin{array}{l}\text { Multi-locus } \\
\text { sequence typing } \\
\text { (MLST) }\end{array}$ & Plasmids names & $\begin{array}{l}\text { Percent identity against } \\
\text { plasmid }\end{array}$ \\
\hline S1 & 99 & $5,817,465$ & $1,00,468$ & 56.63 & 14 & $\begin{array}{l}\text { ColKP3, IncFIB(K), } \\
\text { IncFIB(Mar), IncFII, IncHI1B, } \\
\text { IncR }\end{array}$ & $100,100,99,100,100,100$ \\
\hline S2 & 53 & $5,166,762$ & $8,02,570$ & 57.35 & 11 & $\begin{array}{l}\text { IncA/C2, IncFIB(pQil), } \\
\text { IncFII(K) }\end{array}$ & $100,100,100$ \\
\hline S3 & 87 & $5,709,294$ & $1,85,631$ & 56.96 & 147 & $\begin{array}{l}\text { Col440I, ColRNAl, } \\
\text { IncFIB(pQil), IncFII(K), } \\
\text { IncL/M(pOXA-48), IncR, } \\
\text { IncX4 }\end{array}$ & $96,96,96,97,100,100,100$ \\
\hline S4 & 88 & $5,699,223$ & $1,67,147$ & 56.72 & 37 & $\begin{array}{l}\text { ColKP3, FIA(pBK30683), } \\
\text { IncFII(K), IncHI1B }\end{array}$ & $100,97,95,99$ \\
\hline S5 & 254 & $5,993,514$ & $1,00,468$ & 56.63 & 147 & $\begin{array}{l}\text { Col440I, ColKP3,CoIRNAI, } \\
\text { IncFIB(pKPHS1, IncFIB(pQil), } \\
\text { IncFII(K), IncL/M(pOXA-48), } \\
\text { IncR, IncX4 }\end{array}$ & $\begin{array}{l}96,100,96,98,100,97,100 \\
100,100\end{array}$ \\
\hline S6 & 72 & $5,758,675$ & $2,98,382$ & 56.99 & 147 & $\begin{array}{l}\text { ColRNAl, IncFIB(pQil), } \\
\operatorname{lncFII(K),~IncL/M(pOXA-48),~} \\
\text { IncR }\end{array}$ & $96,100,97,100,100$ \\
\hline S7 & 97 & $5,408,166$ & $2,59,061$ & 57.33 & 39 & $\begin{array}{l}\text { Col440I, IncFIB(pQil), } \\
\text { IncFII(K) }\end{array}$ & $100,100,97$ \\
\hline S8 & 122 & $5,742,261$ & $2,63,702$ & 56.95 & 17 & $\begin{array}{l}\operatorname{IncFIB(K),~IncFIB(pKPHS1),~} \\
\operatorname{IncFIB(pQil),~IncFII(K),~IncX3~}\end{array}$ & $99,98,100,97,100$ \\
\hline
\end{tabular}

No. of contigs number of contiguous data; N50 sequence length at 50\%, GC content glycine cystine content; MLST multi-locus sequence typing. The most common MLST sequence strain isolated was ST147 $(n=3)$

\section{Phylogenetic analysis}

The dendrogram was plotted $(n=41,172$ as per 6 th March 2021) using NCBI Pathogen Detection database (Fig. 1). S3, S5 and S6 (ST147) did not cluster with the reference strain, however, S4 (ST37) was the closest, followed by S2 (ST11) and then S1 (ST14) and S8 (ST17).
In addition to the above, clustering with other sequences deposited in the database was also observed. S4 clustered with clinical strain CFSAN059641 isolated in 2020 in Pakistan and also with the other clinical strains deposited (PDT0009285111). S1 clustered with

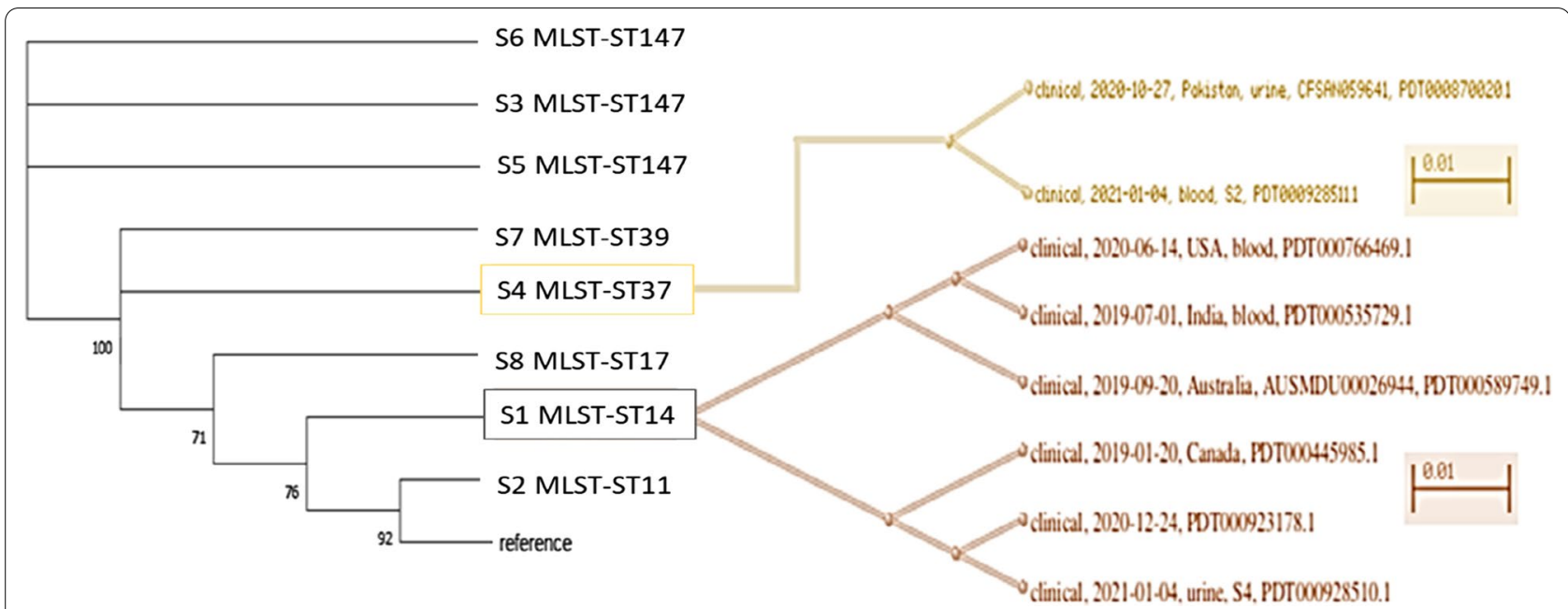

Fig. 1 Dendrogram colistin-resistant—Klebsiella pneumoniae isolates, along with magnified sub-clades for S1 and S4 based on SNP cluster was plotted using Pathogen Detection Browser 
clinical strains isolated in USA (2020), and also with others from Australia, Canada and India (2019).

\section{Discussion}

Although novel treatment approaches are being explored [20-23], increasing prevalence of colistin resistant $K$. pneumoniae, continue to pose a serious global threat.

In Pakistan $m c r-1$ gene has been detected in $23.3 \% E$. $\operatorname{coli}(\mathrm{n}=120)$ and $40 \%$ of K. pneumoniae $(\mathrm{n}=60)$ [24]. Our study however revealed absence of $m c r-1$ gene in all isolates tested. These findings are consistent with earlier studies also reporting absence of $m c r-1$ gene in colistin resistant clinical study isolates [25] and suggest presence of factors other than $m c r-1$ gene contributing to colistin resistance in these isolates.

Alternative mechanisms suggested for colistin resistance include: decreased drug permeability by porin loss, resistance to antibiotic penetration through bio-film formation [26] and mutations leading to alteration in antibiotic binding sites and efflux pump [27]. In our isolates WGS showed the modifications in PagP and PhoR genes of lipid A pathway. Mutations in PagP has been shown to be associated with colistin resistance [28, 29]. Also, mutations in the phoB-phoR operon have shown to contribute to the anti-microbial resistance by downregulation of PhoE [30]. Mutations found in genes of Lipid A-Ara4N pathway including Arn_DH/F, ArnC, ArnT have been reported in K. pneumonia [28]. We additionally detected mutations in $U g d H$ gene of Lipid A-Ara4N pathway which has been shown to be implicated in colistin resistance in E. coli [31]. Gram negative bacteria develop resistance against cationic antimicrobial peptides by masking negative charges of the lipid A phosphate substituent through the addition of L-Ara4N positively charged-moieties. Briefly, L-Ara4N is transferred to lipid A by a lipid carrier in a reaction catalysed by ArnT. The synthesis of lipid carrier linked to L-Ara4N is catalyzed by UDP-Glc 6-dehydrogenase (Ugd), ArnA, ArnB, ArnC, and ArnD [32]. This pathway is well-explained in studies in E. coli and S. enterica sv. typhimurium [33]. It is important to note that the mutations found in Lipid A and $\mathrm{L}-\mathrm{Ara} 4 \mathrm{~N}$ pathway were present in all isolates comprising of 6 colistin resistant and 2 colistin intermediate strains. One of our colistin intermediate isolate with mutations in both Lipid A and A-Ara4N pathway, failed to achieve microbiological clearance over 3 years, despite treatment. There is inherent variability in testing methods for colistin susceptibility, hence the susceptible category has been removed by CLSI [14]. Colistin monotherapy is also discouraged to avoid excessive reliance on even the currently recommended broth dilution susceptibility testing method. Hence, learning more about molecular mechanisms of colistin resistance might be helpful for taking clinical decisions in future.

The MLST done showed that eight $K$. pneumoniae isolates belonged to 6 different sequence types (ST11, ST14, ST17, ST37, ST39 and ST147) hence pointing towards the presence of considerable genetic diversity among them. Previous studies have shown that ST11 and ST14 have been detected amongst clinical isolates from Pakistan [25]. Globally, ST11 has been reported in Korea [34], Tunisia [35], and Egypt and linked with the presence of CTX-M gene [3]. ST 14 has been shown to be circulating in Dubai [36], India [37], Pakistan [25] and described to carry NDM-1 and CTX-M [38]. ST 17 are also reported to be mostly extended spectrum beta lactamases (ESBL) carrying clones [39]. ST 147 detected has been shown globally to be linked with resistance [15, 40, 41]. ST37 has been reported with carbapenem resistance and ST39 [42] with ESBLs.

This study highlights the significant challenges posed by multi-drug resistant $K$. pneumoniae strains to global health and emphasizes the need to identify factors contributing to resistance towards their better treatment and control.

\section{Limitations}

Although the study highlights mechanism of colistin resistance alternate to $m c r-1$ gene in clinical isolates in Pakistan, it can be further strengthened by increasing sample size and by performing functional studies to validate the role of mutations found in Lipid A and L-Ara4N pathway in contributing colistin resistance.

\section{Abbreviations}

DNA: Deoxyribonucleic acid; ESBL: Extended spectrum beta lactamases; LPS: Lipopolysaccharide; MDR: Multi drug resistant; $m c r 1$ : Mobile colistin resistance 1; MLST: Multi-locus sequence typing; NCTC: National collection of type culture collection strain; WGS: Whole genome sequencing.

\section{Supplementary Information}

The online version contains supplementary material available at https://doi. org/10.1186/s13104-021-05867-3.

Additional file 1: Table S1. Primers and probe sequences to target the plasmid-mediated colistin resistance ( $\mathrm{mcr}$ - 1 ) and housekeeping gene $r$ o. Figure S1. Amplification of $\mathrm{mcr}-1$ gene in a positive control NCTC $13846 \mathrm{E}$. coli strain. DNA was extracted from the pure culture of NCTC 13846 E. coli strain using DNA extraction kit (Qiagen) as per manufacturers instruction. Extracted DNA was subjected to PCR using sequence specific primers of mcr-1 gene. The figure shows the amplification of $\mathrm{mcr}-1$ gene in the positive control used in the experiment. PC denotes positive control and NTC denotes non-template control.

\section{Acknowledgements}

We would like to thank Health Security Partners (HSP)-USA for funding this project. We would also like to thank the staff of Department of Pathology and Laboratory Medicine for providing help during the project. 


\section{Authors' contributions}

Conception and design: $\mathrm{RH}, \mathrm{KI}, \mathrm{SU}, \mathrm{JF}, \mathrm{ZH}, \mathrm{SS}, \mathrm{JR}$; analysis and interpretation: $\mathrm{KI}$, $\mathrm{SU}, \mathrm{ZH}, \mathrm{SAR}, \mathrm{NJ}$; drafting the manuscript for important intellectual content: $\mathrm{RH}$, $\mathrm{KI}, \mathrm{SU}, \mathrm{JF}, \mathrm{ZH}$. All authors read and approved the final manuscript.

\section{Funding}

This study was supported by the Health Security Partners (HSP)-USA, as part of their collaboration with the Department of Pathology and Laboratory Medicine, Aga Khan University, for laboratory capacity building and strengthening for antimicrobial resistance surveillance. We declare that there was no competing interest by the funding agency in determining or influencing the study at any point.

\section{Availability of data and materials}

The datasets used and/or analyzed during the current study are available from the corresponding author on reasonable request.

\section{Declarations}

\section{Ethics approval and consent to participate}

The clinical isolates from the Aga Khan University Clinical Laboratory strain bank stored at $-80^{\circ} \mathrm{C}$ were used anonymously. No samples from human subjects were directly used. Hence, the need from obtaining informed consent from human subjects was waived and this study received exemption from full ethical review by The Aga Khan University's Institutional Ethical Review Committee (ERC number: 2018-0424-813)

\section{Consent for publication}

Not applicable.

\section{Competing interests}

Nothing to disclose.

\section{Author details}

'Department of Pathology and Laboratory Medicine, Aga Khan University, Karachi, Pakistan. ${ }^{2}$ Department of Infection Biology, Faculty Infectious and Tropical Diseases, London School of Hygiene and Tropical Medicine, London, UK. ${ }^{3}$ Health Security Partners, Washington, DC 20009, USA.

Received: 29 September 2021 Accepted: 29 November 2021 Published online: 14 December 2021

\section{References}

1. Behzadi P, Garcia-Perdomo HA, Karpinski TM, Issakhanian L. Metallo-sslactamases: a review. Mol Biol Rep. 2020;47(8):6281-94.

2. Reyes J, Aguilar AC, Caicedo A. Carbapenem-resistant Klebsiella pneumoniae: microbiology key points for clinical practice. Int J Gen Med. 2019;12:437-46

3. Woodford N, Zhang J, Warner M, Kaufmann ME, Matos J, Macdonald A, et al. Arrival of Klebsiella pneumoniae producing KPC carbapenemase in the United Kingdom. J Antimicrob Chemother. 2008;62(6):1261-4

4. Wang R, van Dorp L, Shaw LP, Bradley P, Wang Q, Wang X, et al. The global distribution and spread of the mobilized colistin resistance gene mcr-1. Nat Commun. 2018;9(1):1179.

5. Zhou K, Cattoir V, Xiao Y. Intrinsic colistin resistance. Lancet Infect Dis. 2016;16(11):1227-8.

6. Liu YY, Wang Y, Walsh TR, Yi LX, Zhang R, Spencer J, et al. Emergence of plasmid-mediated colistin resistance mechanism $\mathrm{mcr}-1$ in animals and human beings in China: a microbiological and molecular biological study. Lancet Infect Dis. 2016;16(2):161-8.

7. Mohsin M, Raza S, Roschanski N, Guenther S, Ali A, Schierack P. Description of the first Escherichia coli clinical isolate harboring the colistin resistance gene $\mathrm{mcr}-1$ from the Indian subcontinent. Antimicrob Agents Chemother. 2017. https://doi.org/10.1128/AAC.01945-16.

8. Falagas ME, Rafailidis PI, Matthaiou DK. Resistance to polymyxins: mechanisms, frequency and treatment options. Drug Resist Updat. 2010;13(4-5):132-8
9. Velkov T, Deris ZZ, Huang JX, Azad MA, Butler M, Sivanesan S, et al. Surface changes and polymyxin interactions with a resistant strain of Klebsiella pneumoniae. Innate Immun. 2014;20(4):350-63.

10. Cheng HY, Chen YF, Peng HL. Molecular characterization of the PhoPQPmrD-PmrAB mediated pathway regulating polymyxin $B$ resistance in Klebsiella pneumoniae CG43. J Biomed Sci. 2010;17:60.

11. Poirel L, Jayol A, Bontron S, Villegas MV, Ozdamar M, Turkoglu S, et al. The mgrB gene as a key target for acquired resistance to colistin in Klebsiella pneumoniae. J Antimicrob Chemother. 2015:70(1):75-80.

12. Wright MS, Suzuki Y, Jones MB, Marshall SH, Rudin SD, van Duin D, et al. Genomic and transcriptomic analyses of colistin-resistant clinical isolates of Klebsiella pneumoniae reveal multiple pathways of resistance. Antimicrob Agents Chemother. 2015;59(1):536-43.

13. CLSI. Performance Standards for Antimicrobial Susceptibility Testing. 30th ed. CLSI supplement M100. Wayne, PA: Clinical and Laboratory Standards Institute; 2020

14. Wayne P. Methods for dilution antimicrobial susceptibility tests for bacteria that grow aerobically. CLSI document. Wayne: Clinical and Laboratory Standards Institute; 2019. p. M07-A10.

15. Sato T, Harada K, Usui M, Tsuyuki Y, Shiraishi T, Tamura Y, et al. Tigecycline susceptibility of Klebsiella pneumoniae complex and Escherichia coli isolates from companion animals: the prevalence of tigecycline-nonsusceptible K. pneumoniae complex, including internationally expanding human pathogenic lineages. Microb Drug Resist. 2018;24(6):860-7.

16. Chabou S, Leangapichart T, Okdah L, Le Page S, Hadjadj L, Rolain JM. Real-time quantitative PCR assay with Taqman ${ }^{\circledR}$ probe for rapid detection of mcr-1 plasmid-mediated colistin resistance. New Microbes New Infect. $2016 ; 13: 71-4$

17. Gomes AEI, Stuchi LP, Siqueira NMG, Henrique JB, Vicentini R, Ribeiro ML, et al. Selection and validation of reference genes for gene expression studies in Klebsiella pneumoniae using reverse transcription quantitative real-time PCR. Sci Rep. 2018;8(1):9001.

18. Diancourt L, Passet V, Verhoef J, Grimont PA, Brisse S. Multilocus sequence typing of Klebsiella pneumoniae nosocomial isolates. J Clin Microbiol. 2005:43(8):4178-82.

19. Ahmadi M, Ranjbar R, Behzadi P, Mohammadian T. Virulence factors, antibiotic resistance patterns, and molecular types of clinical isolates of Klebsiella Pneumoniae. Expert Rev Anti Infect Ther. 2021. https://doi.org/ 10.1080/14787210.2022.1990040.

20. Le Trong N, Viet Ho D, Quoc Doan T, Le Tuan A, Raal A, Usai D, et al. Biological activities of essential oils from leaves of Paramignya trimera (Oliv.) Guillaum and Limnocitrus littoralis (Miq.) Swingle. Antibiotics. 2020. https://doi.org/10.3390/antibiotics9040207.

21. Donadu MG, Le Trong N, Viet Ho D, Quoc Doan T, Le Tuan A, Raal A, et al. Phytochemical compositions and biological activities of essential oils from the leaves, rhizomes and whole plant of Hornstedtia bella Skornick. Antibiotics. 2020. https://doi.org/10.3390/antibiotics9060334.

22. Bua A, Usai D, Donadu MG, Delgado Ospina J, Paparella A, ChavesLopez C, et al. Antimicrobial activity of Austroeupatorium inulaefolium (H.B.K.) against intracellular and extracellular organisms. Nat Prod Res. 2018:32(23):2869-71.

23. Cannas S, Usai D, Pinna A, Benvenuti S, Tardugno R, Donadu M, et al. Essential oils in ocular pathology: an experimental study. J Infect Dev Ctries. 2015;9(6):650-4.

24. Hameed F, Khan MA, Bilal H, Muhammad H, Tayyab Ur R. Detection of mcr-1 gene in multiple drug resistant Escherichia coli and Klebsiella pneumoniae in human clinical samples from Peshawar, Pakistan. Comb Chem High Throughput Screen. 2020. https://doi.org/10.2174/1386207323 666200914100119.

25. Lomonaco S, Crawford MA, Lascols C, Timme RE, Anderson K, Hodge DR, et al. Resistome of carbapenem- and colistin-resistant Klebsiella pneumoniae clinical isolates. PLoS ONE. 2018;13(6):e0198526.

26. Reza A, Sutton JM, Rahman KM. Effectiveness of efflux pump inhibitors as biofilm disruptors and resistance breakers in gram-negative (ESKAPEE) bacteria. Antibiotics. 2019. https://doi.org/10.3390/antibiotics8040229.

27. Usai D, Donadu M, Bua A, Molicotti P, Zanetti S, Piras S, et al. Enhancement of antimicrobial activity of pump inhibitors associating drugs. J Infect Dev Ctries. 2019;13(2):162-4.

28. Mathur P, Veeraraghavan B, Devanga Ragupathi NK, Inbanathan FY, Khurana S, Bhardwaj N, et al. Multiple mutations in lipid-A modification 
pathway and novel fosA variants in colistin-resistant Klebsiella pneumoniae. Future Sci OA. 2018;4(7):FSO319.

29. Sun L, Rasmussen PK, Bai Y, Chen X, Cai T, Wang J, et al. Proteomic changes of Klebsiella pneumoniae in response to colistin treatment and crrB mutation-mediated colistin resistance. Antimicrob Agents Chemother. 2020. https://doi.org/10.1128/AAC.02200-19.

30. Bialek-Davenet S, Mayer N, Vergalli J, Duprilot M, Brisse S, Pages JM, et al. In-vivo loss of carbapenem resistance by extensively drug-resistant Klebsiella pneumoniae during treatment via porin expression modification. Sci Rep. 2017;7(1):6722.

31. Breazeale SD, Ribeiro AA, Raetz CR. Oxidative decarboxylation of UDP-glucuronic acid in extracts of polymyxin-resistant Escherichia coli. Origin of lipid a species modified with 4-amino-4-deoxy-L-arabinose. J Biol Chem. 2002;277(4):2886-96.

32. King JD, Kocincova D, Westman EL, Lam JS. Review: lipopolysaccharide biosynthesis in Pseudomonas aeruginosa. Innate Immun. 2009;15(5):261-312

33. Yan A, Guan Z, Raetz CR. An undecaprenyl phosphate-aminoarabinose flippase required for polymyxin resistance in Escherichia coli. J Biol Chem. 2007;282(49):36077-89

34. Choi M, Ko KS. Identification of genetic alterations associated with acquired colistin resistance in Klebsiella pneumoniae isogenic strains by whole-genome sequencing. Antibiotics. 2020. https://doi.org/10.3390/ antibiotics9070374.

35. Jaidane N, Bonnin RA, Mansour W, Girlich D, Creton E, Cotellon G, et al. Genomic insights into colistin-resistant Klebsiella pneumoniae from a Tunisian teaching hospital. Antimicrob Agents Chemother. 2018. https:// doi.org/10.1128/AAC.01601-17.

36. Moubareck CA, Mouftah SF, Pal T, Ghazawi A, Halat DH, Nabi A, et al. Clonal emergence of Klebsiella pneumoniae ST14 co-producing OXA-48type and NDM carbapenemases with high rate of colistin resistance in Dubai, United Arab Emirates. Int J Antimicrob Agents. 2018;52(1):90-5.

37. Shankar C, Venkatesan M, Rajan R, Mani D, Lal B, Prakash JAJ, et al. Molecular characterization of colistin-resistant Klebsiella pneumoniae and its clonal relationship among Indian isolates. Indian J Med Res. 2019;149(2):199-207.

38. Giske CG, Froding I, Hasan CM, Turlej-Rogacka A, Toleman M, Livermore D, et al. Diverse sequence types of Klebsiella pneumoniae contribute to the dissemination of blaNDM-1 in India, Sweden, and the United Kingdom. Antimicrob Agents Chemother. 2012;56(5):2735-8.

39. Navon-Venezia S, Kondratyeva K, Carattoli A. Klebsiella pneumoniae: a major worldwide source and shuttle for antibiotic resistance. FEMS Microbiol Rev. 2017;41(3):252-75.

40. Sonnevend A, Ghazawi A, Hashmey R, Haidermota A, Girgis S, Alfaresi $\mathrm{M}$, et al. Multihospital occurrence of pan-resistant Klebsiella pneumoniae sequence type 147 with an ISEcp1-directed blaOXA-181 insertion in the mgrB gene in the United Arab Emirates. Antimicrob Agents Chemother. 2017. https://doi.org/10.1128/AAC.00418-17.

41. Mathers AJ, Stoesser N, Chai W, Carroll J, Barry K, Cherunvanky A, et al. Chromosomal integration of the Klebsiella pneumoniae carbapenemase gene, blaKPC, in Klebsiella species is elusive but not rare. Antimicrob Agents Chemother. 2017. https://doi.org/10.1128/AAC.01823-16.

42. Belbel Z, Chettibi H, Dekhil M, Ladjama A, Nedjai S, Rolain JM. Outbreak of an armA methyltransferase-producing ST39 Klebsiella pneumoniae clone in a pediatric Algerian Hospital. Microb Drug Resist. 2014;20(4):310-5.

\section{Publisher's Note}

Springer Nature remains neutral with regard to jurisdictional claims in published maps and institutional affiliations. 